\title{
Entorno y cuidado: circunstancias que influyen en la calidad de la atención médica percibida por los colombianos
} Environment and care: circumstances that influence the quality of
healthcare perceived by the people in Colombia Ambiente e cuidado: circunstâncias que influenciam a qualidade
da atenção médica percebida pelos colombianos

Jessica Andrea Duque-Castro, Est.*

Juliana Ortiz-Urbano, Est. *

Diana Marcela Rengifo-Arias, Enf., MSc.**

\section{Resumen}

Introducción. El análisis de las situaciones de salud, en el acto del cuidado de la enfermería, requiere de conocimientos, pues es el marco conceptual y la guía de esta labor. Aunque el cuidado integral de la salud, según Watson, depende del compromiso moral del enfermero de preservar la dignidad humana; son frecuentes los informes que muestran una mala percepción de la prestación de servicios de salud. En los informes, se indica que el $36 \%$ de los encuestados tienen una mala o muy mala percepción de la calidad de la atención médica. El objetivo es reflexionar sobre las circunstancias que influyen en la calidad de la atención médica percibida por los colombianos. Temas de reflexión. Se abordará el tema desde los patrones de conocimiento de la enfermería: el empírico, el estético, el ético, el sociocultural y el personal. Discusión. Las condiciones que influyen en la percepción de la calidad de la atención médica se agrupan en dos conceptos del metaparadigma de la enfermería. El primero es el entorno, en el que existen barreras de oferta y demanda, como la tecnificación, la infraestructura y los recursos del sistema de salud. El segundo es el cuidado, en el que la condición laboral tiene incidencia, ya que los profesionales de enfermería sienten culpabilidad, agotamiento y estrés, y comprometen la buena percepción del cuidado médico y el sentido de trascendencia profesional. Conclusiones. El acto del cuidado de la enfermería está rodeado de situaciones poco favorables, dadas las barreras en el sistema de salud colombiano, que comprometen la labor del enfermero. Por lo tanto, es necesario reflexionar y desarrollar conceptos relacionados con los patrones de conocimiento de la disciplina, que inciden en la formación de los nuevos profesionales. Esto, con el

* Estudiante de Enfermería, Universidad Alexander Von Humboldt, Armenia, Quindío, Colombia.

** Enfermera, Magíster en Enfermería con énfasis en el cuidado de la Salud Cardiovascular, Magíster en Nutrición, Obesidad y Técnicas Culinarias, Docente Corporación Universitaria Empresarial Alexander von Humboldt, Armenia, Quindío, Colombia. 
fin de cumplir a cabalidad el deber ser y el hacer de la enfermería en Colombia. [Duque-Castro JA, OrtizUrbano J, Rengifo-Arias DM. Entorno y cuidado: circunstancias que influyen en la calidad de la atención percibida por los colombianos. MedUNAB. 2018;21(2):60-68. doi:10.29375/01237047.2799]

Palabras clave: Atención de la Enfermería; Condiciones de Trabajo; Garantía de la Calidad de la Atención Médica; Humanización de la Atención y los Servicios de Enfermería

\section{Abstract}

Introduction. The analysis of health situations, in terms of nursing care, requires knowledge, since it is the conceptual framework and guide for this work. Although comprehensive healthcare, according to Watson, depends on the nurse's moral commitment to preserve human dignity, there are frequent reports that show a poor perception of the provision of healthcare services. The reports indicate that $36 \%$ of those surveyed had a poor or very poor perception of the quality of healthcare. The objective is to reflect on the circumstances that influence the quality of healthcare perceived by the people in Colombia. Topics of reflection. The topic will be addressed through the patterns of knowledge of nursing: empirical, aesthetic, ethical, sociocultural and personal. Discussion. The conditions that influence the perception of healthcare quality are grouped into two nursing metaparadigm concepts. The first is the environment, in which there are supply and demand barriers, such as technification, infrastructure and resources of the healthcare system. The second is the care, on which the employment status has an influence, since nursing professionals feel guilt, exhaustion and stress, and compromise the good perception of healthcare and the sense of professional importance. Conclusions. The act of caring in nursing is surrounded by unfavorable situations, given the barriers in the Colombian healthcare system that compromise the work of nurses. Therefore, it is necessary to reflect and develop concepts related to the patterns of knowledge of this discipline, which have an effect on the training of new professionals. This is in order to fully comply with the duty and practice of nursing in Colombia. [Duque-Castro JA, OrtizUrbano J, Rengifo-Arias DM. Environment and care: circumstances that influence the quality of medical care perceived by the people in Colombia. MedUNAB. 2018;21(2):60-68. doi:10.29375/01237047.2799]

Keywords: Nursing Care; Employment Status;
Guarantee of Healthcare Quality; Humanization of Care and Nursing Services

\section{Resumo}

Introdução. A análise das situações de saúde, no ato do cuidado da enfermagem, requer conhecimento, pois é o referencial conceitual e o guia desta atividade. Embora os cuidados integrais de saúde, segundo Watson, dependem do compromisso moral do enfermeiro para preservar a dignidade humana; há relatos frequentes que mostram uma má percepção da prestação de serviços de saúde. Nos relatórios, indica-se que 36\% dos entrevistados têm uma percepção ruim ou muito ruim da qualidade do atendimento médico. O objetivo é refletir sobre as circunstâncias que influenciam a qualidade da atenção médica percebida pelos colombianos. Temas para reflexão. $O$ tema será abordado a partir dos padrões de conhecimento da enfermagem: o empírico, o estético, o ético, o sociocultural e o pessoal. Discussão. As condições que influenciam a percepção da qualidade da atenção médica são agrupadas em dois conceitos do metaparadigma da enfermagem. O primeiro é o ambiente, no qual existem barreiras de oferta e demanda, como a tecnificação, a infraestrutura e os recursos do sistema de saúde. O segundo é o cuidado, no qual a condição de trabalho tem impacto, uma vez que os profissionais de enfermagem sentemse culpados, exaustos e estressados, comprometendo a boa percepção do cuidado médico e o sentido de transcendência profissional. Conclusões. $\mathrm{O}$ ato do cuidado de enfermagem está cercado de situações desfavoráveis, dadas as barreiras no sistema de saúde colombiano, que comprometem o trabalho do enfermeiro. Portanto, é necessário refletir e desenvolver conceitos relacionados aos padrões de conhecimento da disciplina, que afetam a formação dos novos profissionais. Isso, a fim de cumprir integralmente o dever de ser e exercer a enfermagem na Colômbia. [Duque-Castro JA, Ortiz-Urbano J, Rengifo-Arias DM. Ambiente e cuidado: circunstâncias que influenciam a qualidade da atenção médica percebida pelos colombianos. MedUNAB. 2018;21(2):60-68. doi:10.29375/01237047.2799]

Palavras-chave: Cuidados de Enfermagem; Condições de Trabalho; Garantia da Qualidade dos Cuidados de Saúde; Humanização dos Cuidados e Serviços de Enfermagem 


\section{Introducción}

El ser humano es dinámico, flexible y cambiante. Este dinamismo determina e individualiza el cuidado, un fenómeno universal e intercultural. El desarrollo de los conocimientos de la enfermería debe responder a cabalidad con esta individualización del cuidado; por lo que es insostenible crear un conocimiento generalizado y de aplicabilidad estándar a todas las personas.

Durante la evolución histórica de la enfermería, el profesional ha desarrollado un proceso teórico y metodológico que le permite identificar situaciones vitales que requieren del cuidado de la enfermería. Esto, con el fin de promover la salud, prevenir la enfermedad, y realizar acompañamiento durante la recuperación y la muerte. También tiene el objetivo de lograr el desarrollo de elementos básicos para comprender y aplicar los patrones de conocimiento de la disciplina y el proceso de atención de la enfermería como operacionalización del método científico (1). Formular un cuerpo de conocimientos y emplearlo en el día a día permite diferenciar un profesional de enfermería experto de aquel que solo sigue las normas y se orienta por las tareas que debe realizar. Esto otorga seguridad en el actuar y fortalece la autoimagen profesional (2).

Sidani e Irvine describen tres tipos de roles que el profesional de enfermería ejerce: independiente, dependiente e interdependiente. El primero se relaciona con el desarrollo de funciones específicas de los enfermeros (cuidado de la salud); el segundo, con las actividades asociadas con la implementación de órdenes y tratamientos médicos (3) y el tercero, con el trabajo colaborativo entre los miembros del equipo interdisciplinario para el restablecimiento de la salud. Para garantizar el adecuado cumplimiento de cualquier rol, los enfermeros deben desarrollar prácticas ajustadas a su nivel de formación (3).

El análisis de la situación de salud durante el proceso de atención de enfermería, se fundamenta en teorías y tecnologías propias de la ciencia del cuidado. Según lo establecido en el código deontológico colombiano, Ley 911, el acto de cuidado de la enfermería tiene como propósito desarrollar las potencialidades individuales y colectivas. Para ello, el profesional de enfermería emplea conocimientos propios y de las ciencias biológicas, sociales y humanísticas, que le permiten promover una comunicación y relación humana en pro del cuidado; esto es el ser y la esencia de la profesión. El cuidado implica un juicio de valor y un proceso dinámico y participativo, en el que se identifican y priorizan las necesidades que deben ser atendidas en el plan de cuidados (4). Esto se hace a partir de juicios reflexivos, que surgen de procesos de metacognición, en los que se tienen en cuenta conceptos de la enfermería, como las interacciones entre la persona, el cuidado, el entorno y la salud, y los patrones de conocimiento de la disciplina: conocimiento empírico, sociocultural, ético, estético y personal.

La ciencia de la enfermería se fundamenta en el estudio de las experiencias del cuidado que desarrollan los seres humanos (5). Cada concepto es una pieza clave para comprender las necesidades humanas (dinámicas, propias y particulares de cada persona) con las cuales se interactúa durante el ejercicio profesional (6). Por esto, es importante reconocer la diferencia entre la atención de la enfermería (que involucra aspectos técnicos y procedimentales) y el cuidado de enfermería, que permite la construcción de relaciones e imaginarios sociales entorno a la percepción del cuidado. Esta percepción debe ser entendida como un proceso mental, en el que el paciente y el profesional identifican los comportamientos del cuidado conforme a sus criterios, valores, vivencias, experiencias y expectativas, y al entorno situacional y al escenario en el que se da el acto del cuidado (7).

La humanización en la atención en salud se ha convertido en política de Estado. Según esta política, humanizar es un asunto ético relacionado con los valores que rigen la conducta y las relaciones en el sistema de salud, y en el que se enfatiza la dignidad de todo ser humano (6). Sin embargo, son comunes los informes sobre la percepción inadecuada que hay de la prestación de servicios de salud y la poca satisfacción de la atención recibida. Para el Departamento Nacional de Planeación (DNP) la mala percepción y la poca satisfacción se deben a las barreras que hay para acceder a los servicios de salud. Por ejemplo, hay una amplia cobertura de las IPS (Instituciones Prestadoras de Servicios de Salud), un $56 \%$ de infraestructura, en las principales ciudades (Bogotá, Medellín y Cali), pero poca cobertura en ciudades como la Guajira, Chocó y Vichada, en las que hay: "1.53 camas disponibles en servicios de hospitalización, 1.31 camas de cuidado intensivo, 0.11 de cuidado intermedio y 0.14 de cuidado intensivo, 0.09 ambulancias, 0.07 ambulancias básicas y 0.02 medicalizadas por cada 1000 habitantes" (8).

Las demoras relacionadas con la tramitología de las IPS son otra barrera para acceder a los servicios de salud. Según el Informe Nacional de Calidad de la 
Atención en Salud del 2015, el período de espera para la asignación de citas con médico general pasó de dos a tres días, con especialistas, de siete a doce días y con medicina interna, de nueve a doce días. Por otro lado, la Encuesta de Evaluación de los Servicios de las EPS 2013-2014 arrojó que la mayoría de los usuarios encuestados se encontraran inconformes con los tiempos de espera, además de indicar que el $46 \%$ de los usuarios consideraron difícil acceder a los servicios de salud de su EPS (8); coincidiendo con el estudio de Arrivillaga et al., quienes indicaron una percepción de mala y muy mala calidad de la atención hasta en un 36\% (9), entendiendo la calidad de la atención en salud como "la provisión de servicios accesibles y equitativos con un nivel profesional óptimo, que tiene en cuenta los recursos disponibles y logra la adhesión y satisfacción del usuario" (10). En este mismo estudio del 2016, se demostró que la asignación de citas de consulta de medicina general y con especialista había presentado demoras, en especial, en el tiempo de autorización por parte de las EPS y de los médicos especialistas. Por otro lado, el acceso a los medicamentos también se vio comprometido, pues el $25 \%$ de los encuestados reportaron problemas o bien porque no se los habían entregado a tiempo o bien porque no estaban incluidos en el Plan Obligatorio de Salud (9).

Asimismo, la falta de información durante los procedimientos también influyó en la mala percepción de la atención médica, pues generó un sentimiento de deshumanización. A esto hay que añadirle la falta de comunicación y relaciones efectivas, pues los funcionarios no atendieron las solicitudes. Así, Rodríguezetal. evidenciaron en su artículo expresiones como: "Con Derechos de Petición o Acción de Tutela (AT) es que atienden rápido, aun con la AT se violan derechos. Hubo un caso de desistimiento: Desisto de la atención, para evitar tanto obstáculo y permisos en el trabajo, a pesar de sentirme enferma" (11).

Por esta razón, en el contexto colombiano se ha venido desarrollando durante los últimos años y cada vez con mayor fuerza el concepto de "humanización de la atención en salud" (6). Sin embargo, resulta paradójico que el concepto de humanización, entendido como "un proceso de comunicación y apoyo mutuo entre las personas, encausada hacia la transformación y comprensión del espíritu esencial de la vida" (12) que, según Guerrero et al., es la filosofía de la enfermería, tenga que ser instaurado mediante la legislación y las políticas de Estado. El hecho de que los protocolos institucionales orienten las relaciones del equipo de salud, y particularmente del grupo de enfermería, con las personas sujeto de cuidado resulta contrario a las pautas teóricas desarrolladas por los académicos dedicados al estudio de la enfermería, como la doctora Jean Watson. Watson (13), en su teoría de Humanización, define el cuidado transpersonal como: "una clase especial de cuidado humano que depende del compromiso moral de la enfermera, de proteger y realzar la dignidad humana", lo que permite dar trascendencia al cuidado humanizado que brinda el profesional de enfermería en su práctica. Por esta razón, cuando se habla de cuidado de la persona se habla de cuidado humano.

¿Qué circunstancias conllevaron a que la humanización se convirtiera en una política de Estado? Podemos decir que una serie de barreras que limitan el trabajo del profesional de enfermería. Entre ellas, la tecnificación, que aunque disminuyó el porcentaje de errores relacionados con la identificación de las personas y el manejo de la historia clínica, en algunos casos, ha relegado el contacto directo con el paciente (mirar las constantes vitales en el monitor ha reemplazado la comprobación de una posible alteración de los signos y síntomas del paciente). Asimismo, la infraestructura de los centros hospitalarios, las precarias condiciones laborales, la carga de trabajo y el aumento de horas no remuneradas minimizan la atención integral en el acto del cuidado, lo que provoca en los profesionales sentimientos de culpabilidad, agotamiento y estrés. Además, el funcionamiento del Sistema de Seguridad Social en Salud colombiano no ha permitido lograr estándares de calidad para la prestación de servicios de salud en algunos lugares del territorio colombiano, puesto que hay una brecha entre la normativa y su ejecución práctica. Esta situación podría comprometer la autonomía y el ejercicio de la enfermería (4), y por consiguiente, generar acciones riesgosas que conlleven a eventos adversos tanto para el personal de salud como para el sujeto de cuidado.

Aunque el quehacer de la enfermería ocurre en un ambiente en el que hay sufrimiento y dolor de los usuarios, las mayores angustias del profesional surgen de las exigencias relacionadas con la gran carga asistencial. Esto conlleva a que el profesional de enfermería se ocupe de aspectos técnicos y procedimentales que inciden directamente en la calidad del cuidado (11). Los profesionales deben enfocarse en el cumplimiento de objetivos medibles en la práctica clínica; situación favorable para la institución, pero desfavorable para el gremio, que debe hacer una gran cantidad de tareas administrativas relegando el cuidado. Esta situación se 
torna paradójica; a la vez que las instituciones buscan calidad en la atención, el profesional de enfermería debe cumplir con la calidad del cuidado (11).

La mano de obra de las instituciones de salud se encuentra conformada en su mayoría por el personal de enfermería. Sin embargo, estos ocupan un lugar secundario y a veces nulo en la ejecución, estructuración y proposición de acciones dirigidas a la salud y de políticas públicas que propenden por un desarrollo social sostenible en materia de atención y servicios de salud (11). Además, estos profesionales presentan en un $88 \%$ de los casos síndrome de Burnout (o síndrome del quemado) a causa del exceso de trabajo. Según Bernal González et al., uno de cada tres profesionales presenta agotamiento emocional, el $18 \%$, despersonalización y el $34 \%$, baja realización personal, lo que provoca que el personal tenga una atención distante y fría con los usuarios. Este ambiente laboral hostil se suma a situaciones relacionadas con las instituciones de salud, como la dificultad de los pacientes y familiares para acceder a los servicios y la falta de recursos - por ejemplo, recursos humanos- (14). Esto, sin duda, afecta la forma en que los profesionales brindan sus servicios a los pacientes: se expresan con apatía y tienen actitudes negativas hacia el trabajo, incluso existe el riesgo de que abandonen sus funciones profesionales. De esta manera, se reducen los servicios de salud disponibles para la población y se aumentan los costos en salud y la deshumanización de la atención; y por lo tanto, se pone en riesgo la eficiencia de la enfermería (13).

Dentro del concepto de calidad asistencial se encuentran tres elementos clave: a) la estructura, que comprende todo lo relacionado a las características materiales y organizacionales de la prestación de servicios, b) el proceso, que se refiere a las actividades que realizan los médicos y trabajadores de la salud durante la atención que brindan a los pacientes y su habilidad para llevarlas a cabo y c) el resultado, que mide los beneficios y el nivel de satisfacción de los pacientes (14). Algunos autores, como Ayala y Barragán, identifican ciertas barreras en el sistema de salud que dependen de algunos elementos claves de este concepto, como por ejemplo, aquellas relacionadas con la oferta (barreras organizacionales e institucionales), como los múltiples trámites para solicitar las citas, la atención ineficaz e inoportuna de los administrativos y el personal de salud, la calidad del servicio y la disponibilidad de recursos humanos y materiales. También hay barreras relacionadas con la demanda, como por ejemplo, los costos de los servicios de salud, la prioridad que se le da al problema de salud presentado por el usuario y los conocimientos del usuario del sistema de salud $(15,16)$.

En el 2016, el Ministerio de Salud y Protección Social Colombiano (MinSalud) mencionó que la oferta y la demanda de los servicios de salud deben adecuarse a los determinantes sociales y las necesidades de salud de cada territorio. Aunque podría decirse que hay un crecimiento en la oferta de salud de forma general, esto no garantiza condiciones adecuadas en el sector. Esta cobertura se ha medido en términos de la cantidad de actividades realizadas, más no en términos de los resultados (de los cambios en las conductas orientadas a la salud); lo que limita la eficacia de las acciones individuales y grupales que tienen como fin la promoción de la salud y la gestión de riesgo. MinSalud, además, observó obsolescencia tecnológica en instituciones de salud de primer nivel, disminución de la capacidad en el segundo nivel y aumento de la capacidad en los de alta complejidad, con una amplia participación de la oferta privada, más que de la oferta pública (17).

El principal problema de salud en Colombia son las Empresas Promotoras de Salud (EPS) propuestas en la Ley 100. Estas aumentaron la inequidad en el servicio y el gasto de mantenimiento, sin mejorar la calidad de la atención. De esta manera, las ganancias adquirieron más importancia que la salud de los colombianos (18), y como consecuencia, los usuarios se vieron afectados. La implementación del Plan Obligatorio de Salud (POS), en el que hay un suministro específico de medicamentos, y los protocolos impuestos por las aseguradoras propiciaron que se quebrantaran los principios éticos de autonomía, beneficencia y no maleficencia, como la obligación de hacer prescripciones con criterio médico, independientemente de los costos de los medicamentos, y comprometieron el cuidado de la enfermería (19).

Actualmente, quienes trabajan en las IPS tienen jornadas laborales extensas, salarios muy bajos y contratos sin prestaciones sociales ni garantías laborales. Los trabajadores han tenido que vincularse a más de dos empleos para tener un salario que les garantice una vida digna (18). Por estas razones, Molina et al (20) afirman que el sistema de salud colombiano no entiende la dignidad humana ni la salud como derechos innatos del ser humano, sino como productos de un mercado. Esto conlleva a que el profesional de enfermería vea cohibida su autonomía por un sistema de trabajo autoritario, que limita su capacidad de decisión. Sin embargo, una adecuada educación ética y moral, y una buena formación científica deberían 
motivar a los profesionales a rehusar la coacción de unos fines empresariales y lucrativos, y proteger la autonomía del gremio y los derechos de los sujetos a quienes se les brinda el cuidado (19).

Por lo expuesto anteriormente, el objetivo del presente artículo es reflexionar sobre las circunstancias que influyen en la percepción de los colombianos de la calidad de la atención y los cuidados médicos.

\section{Temas de Reflexión}

El conocimiento de la enfermería se basa en la comprensión de la naturaleza humana y su respuesta al entorno. Por lo tanto, el profesional de enfermería debe ser capaz de incluir esta perspectiva en los encuentros que desarrolla con el paciente, mediante el intercambio de sus conocimientos y la posibilidad de lo que puede ser descubierto para, finalmente, comprender la necesidad de cuidado de las personas y aprender a cuidar mejor de ellas (20). Para estudiar la disciplina de la enfermería, es necesario comprender el concepto de "conocer" como un acto consciente y una forma particular e individual de visualizar las complejidades de una situación y de reunir los recursos internos experimentados anteriormente para darle significado (21).

El cuerpo de conocimientos en el que se sustenta la racionalidad de la enfermería tiene ciertos patrones, formas y estructuras que caracterizan las maneras en que los profesionales piensan los fenómenos. Bárbara Carper realizó un análisis del contenido conceptual y sintáctico del cuerpo de conocimientos, que en su época fundamentaban a la práctica de Enfermería de la época identificando patrones, formas y estructuras que sirven de horizontes y ejemplifican modos característicos de pensar acerca de los fenómenos (Tabla 1). Carper como profesora de Enfermería, motivada por la necesidad de estructurar el currículum, insiste que entender estos patrones es esencial para la enseñanza y aprendizaje de la enfermería (22), definiendo los patrones de conocimiento disciplinar de la siguiente manera:

1. Patrón de conocimiento empírico (Saber): la Ciencia de Enfermería sustenta el saber obtenido por el estudio de fenómenos por medio de la aplicación del método científico para describir, explicar y predecir fenómenos de interés para la disciplina de Enfermería a fin de controlarlos mediante alguna acción. Este saber se organiza en cuerpos conceptuales y teorías con el propósito de prescribir esos cursos de acción a partir de ese conocimiento, para modificar la realidad, mejorando la calidad de vida de las personas en lo que respecta a su salud (22-24).

2. Patrón de conocimiento estético (del arte): el arte significa todo aquello que se aprendía durante la práctica como experiencia, como aprendizajes asociados a los modelos de imitación y adquisición de conocimiento por acumulación de experiencias no racionales, sin embargo, la dimensión estética del conocimiento de la Enfermería es rasgo de la esencia de la naturaleza de la disciplina (23-25).

3. Patrón de conocimiento ético: en el complejo campo de la salud se deben tomar múltiples decisiones, estas elecciones conducen a preguntas morales fundamentales sobre acciones correctas e incorrectas en todos los ámbitos de la acción profesional: los cuidados directos de Enfermería, la docencia, la gestión, la investigación, la educación para la salud, etc. Dilemas morales que muchas veces no son resueltos por las normas de ética escritas en los códigos profesionales (22 - 25).

4. Patrón de conocimiento personal: toda acción de Enfermería se da a partir de lo que se es, de lo que se desea y de lo que se sabe en una relación interpersonal. Entender y percibir los significados particulares de las experiencias de las personas sobre su proceso de ser saludable, de bienestar, de sufrimiento, de impotencia, de angustia durante la enfermedad y en las transiciones de la vida, implica un acto personal. El uso terapéutico de sí mismo es una meta ampliamente escrita y aceptada en la Enfermería (22 - 25).

5. Patrón de conocimiento socio-cultural: el patrón socio-cultural requiere habilidades de comunicación, movilización y liderazgo que se enmarcan en la participación, las cuales podrían incluirse en el patrón mismo como elementos constitutivos. El patrón de conocimiento socio-cultural al igual que los demás patrones se construyen y desarrolla, a partir de la experiencia, la conciencia y la reflexión, así mismo, es personal y dinámico,; es una experiencia tangible en el mundo y por lo tanto, no es abstracto. Facilita la interacción, en especial, porque pretende entender y modificar el contexto en el que se desarrolla el trabajo del enfermero y de los seres humanos, en general. Por lo tanto, es en este patrón en el que se sustenta el conocimiento de la enfermería y su actuar, pues permite un análisis del micro y el macro entorno, de los recursos y las condiciones que pueden incidir en el acto del cuidado (22 - 25). 
Tabla 1. Caracterización de los patrones de conocimiento de la disciplina

\begin{tabular}{|c|c|}
\hline Patrón de conocimiento & Caracterización \\
\hline Patrón empírico & $\begin{array}{l}\text { Hace referencia a la estructuración y aplicabilidad de los conceptos y teorías de la enfermería } \\
\text { en el contexto y desarrollo de su práctica. Tiene fundamento en la investigación científica } \\
\text { como método para la producción de conocimiento empírico, mediante la observación, la } \\
\text { inspección y la verificación del quehacer profesional; incluye elementos de las ciencias } \\
\text { biológicas, sociales y humanísticas que le permiten fundamentar y desarrollar una relación } \\
\text { de cuidado con una persona multidimensional (24). Este patrón es descriptivo, explicativo, } \\
\text { predictivo, fáctico y verificable; se relaciona con la información, los datos objetivos y lo visible } \\
\text { externamente (23). }\end{array}$ \\
\hline Patrón estético & $\begin{array}{l}\text { Significa hacer lo que se debe hacer, en el momento preciso, logrando que el paciente y el } \\
\text { enfermero tengan certeza de la bondad de la intervención. El estado del arte requiere una } \\
\text { condición de cambio, enmarcada en actitudes, habilidades, destrezas que permitan una } \\
\text { situación de reciprocidad entre la enfermera y la persona sujeto del cuidado (22). }\end{array}$ \\
\hline Patrón ético & $\begin{array}{l}\text { El desarrollo de este componente implica la valoración de la acción mediante elementos } \\
\text { simbólicos, teniendo en cuenta el entendimiento y la creatividad de las acciones del } \\
\text { enfermero. Se visualiza durante el comportamiento del profesional que colabora con } \\
\text { el proceso de valoración, identificación de necesidades y ejecución de los cuidados de } \\
\text { enfermería. Este componente permite al enfermero hacer una valoración propia, que } \\
\text { fundamenta su capacidad de resolución ante dilemas éticos y morales; lo cual hace evidente } \\
\text { la correlación existente entre este patrón y el de conocimiento personal (24). }\end{array}$ \\
\hline Patrón sociocultural & $\begin{array}{l}\text { Un incremento en el conocimiento sociocultural permite entender cuándo y cómo actuar en } \\
\text { relación con los contextos de la enfermería que no solo involucran la práctica, la investigación, } \\
\text { la docencia y la gestión, sino también, los espacios donde se viven las experiencias de salud; } \\
\text { espacios que son influenciados por aspectos culturales, históricos, económicos, sociales y } \\
\text { políticos (22-25). }\end{array}$ \\
\hline \multirow[t]{2}{*}{ Patrón personal } & $\begin{array}{l}\text { Patrón fundamental, debido a su importancia para el entendimiento de la salud en } \\
\text { términos del bienestar y el desarrollo individual, lo que se traduce como el reconocimiento } \\
\text { del enfermero como ser terapéutico. Implica el conocimiento de la experiencia propia } \\
\text { y la comprensión de la disciplina de la enfermería como un proceso que requiere de } \\
\text { interacciones, relaciones y transacciones entre el enfermero y el paciente (25). }\end{array}$ \\
\hline & $\begin{array}{l}\text { Las relaciones interpersonales de la enfermería ya no deben ser pensadas como un don } \\
\text { innato, pues la reciprocidad con los pacientes es el eje del cuidado y demanda tanto } \\
\text { formación con bases científicas como la cognición del profesional práctico que domina la } \\
\text { ciencia y el arte del cuidado (25). }\end{array}$ \\
\hline
\end{tabular}

Fuente: elaboración propia de los autores

6. Patrón de emancipación. Es un conocimiento innovador e imprescindible para el desarrollo de la epistemología y la práctica de la enfermería. Este patrón se define como el logro personal del individuo, al reconocer en la sociedad, su estructura, y política aspectos que inciden en el desarrollo humano. El objetivo de este logro es que el individuo proponga cambios que generen una mejoría en el bienestar de las personas; este patrón incentiva la reflexión, la crítica y el pensamiento creativo para que se formulen propuestas claras que beneficien la salud y el cuidado de las personas $(22-26)$.

Bárbara Carper destacó que los patrones de conocimiento coexisten en el acto del cuidado, es decir, no son excluyentes; por el contrario, se complementan 
e integran la mayoría de las veces. Dan un cimiento a esa aserción que se hace en enfermería respecto a la intención de prestar cuidados integrales a las personas, familias y comunidades. Sin embargo, el actual sistema de salud colombiano, en el que se desenvuelve el profesional de enfermería, genera una barrera para poder expresar y demostrar el potencial de la profesión. A pesar de que son nulas las condiciones mínimas requeridas para el acto del cuidado de la enfermería, los profesionales siguen esforzándose para poder ofrecer una atención digna a los usuarios $(23,24)$.

La acción del enfermero en dicha situación, que surge de una perspectiva crítica y de intervención social, muestra el empoderamiento que tiene el profesional sobre los problemas que aquejan a la sociedad. Si se generan entornos favorables para la ejecución de su función en las instituciones de salud, sin importar el nivel de complejidad, se pueden generar cambios significativos en el actual perfil epidemiológico de la sociedad (26).

\section{Conclusiones}

Las condiciones que influyen en la calidad de la atención y los cuidados se relacionan principalmente con dos conceptos del metaparadigma de la enfermería: entorno y cuidado. En el concepto de entorno se contemplan aspectos relacionados con el sistema de salud colombiano, que presenta barreras relacionadas con la oferta y la demanda de los servicios de salud. Esto compromete las funciones del enfermero y otras situaciones relacionadas con la calidad de la atención.

Las condiciones laborales en las que los profesionales se desempeñan, como la sobrecarga laboral, una baja remuneración y el poco reconocimiento que reciben, influyen en el acto del cuidado y generan riesgo para la salud del enfermero. En consecuencia, hay un impacto indirecto en la capacidad de los profesionales para brindar los cuidados al paciente. Esto hace que se juzgue la eficiencia de los cuidados de la enfermería y haya una percepción desfavorable de los colombianos sobre la humanización de la atención.

Es necesario reflexionar y desarrollar elementos conceptuales relacionados con los patrones de conocimiento de la disciplina, ya que estos inciden en la formación de los nuevos profesionales y las asociaciones académicas. Esto, con el fin de poder desarrollar y cumplir a cabalidad el deber ser y el hacer de la enfermería en Colombia, y de que haya una incidencia indirecta en la calidad de los cuidados.

\section{Conflicto de intereses}

Los autores declaran no tener conflicto de interés.

\section{Referencias}

1. Neumaier-Alves C, Lopes -Corrêa A, Correa-Soares M, Könzgen-Meincke S. El cuidado de enfermería y su evolución histórica: una revisión integrativa. Cul. Cuid. 2015; 19(43): 83-94. doi: 10.14198/cuid.2015.43.09

2. Brykczynski KA. De principiante a experta: excelencia y dominio de la práctica de enfermería clínica. En: Marriner Tomey A, Raile Alligood M, editores. Modelos y teorías en enfermería. Barcelona: Elsevier; 2007. p. 140-166.

3. Laverde-Contreras OL, Carvajal-Carrascal G, Jaimes-Carvajal N, Escobar-Ramírez MC, Arroyo-Marles LP. Rol independiente y ambiente de la práctica de enfermería. Rev Cubana Enfermer [Internet]. 2017 [citado 11 de julio del 2018]; 33(4): [aproximadamente 10p.]. Disponible en: http://www.revenfermeria.sld. cu/index.php/enf/article/view/1013/308

4. Ley 911/2004 del 6 de octubre, por la cual se dictan las disposiciones en materia de responsabilidad deontológica para el ejercicio de la profesión de Enfermería en Colombia (Diario Oficial, número 45.693 del 2004).

5. Borré-Ortiz YM, Lenis-Victoria C, Suárez-Villa M, Tafur-Castillo J. El conocimiento disciplinar en el currículo de enfermería: una necesidad vital para transformar la práctica. Rev. Cienc. Salud. 2015 ; 13(3): 481-491. doi: 10.12804/revsalud13.03.2015.12

6. Bermejo-Higuera JC. ¿Derecho a la humanización? Blog [Internet]. España: José Carlos Bermejo Higuera; 2014 [citado 17 de abril del 2016]; Disponible en: http:/josecarlosbermejo.es/sites/default/ files/articuloes/derecho_a_la_humanizacion.pdf

7. Salgado J, Valenzuela $\bar{S}, \bar{S}$ Sázz K. Comportamientos del cuidado percibidos por estudiantes de enfermería y receptores del cuidado. Cienc. enferm. [Internet]. 2015 [citado 17 de octubre del 2018]; 21(1): 69-79. doi: $10.4067 /$ S0717-95532015000100007

8. Ministerio de Salud y Protección Social. Informe Nacional de Calidad de la Atención en Salud 2015. Bogotá: MINSALUD; 2015. 32p.

9. Arrivillaga M, Aristizabal JC, Pérez M, Estrada VE. Encuesta de acceso a servicios de salud para hogares colombianos. Gac. Sanit. 2016; 30(6): 415-420. doi: 10.1016/j.gaceta.2016.05.008 
10. Cano SM, Giraldo A, Forero C. Concepto de calidad en salud: resultado de las experiencias de la atención, Medellín, Colombia. Rev. Fac. Nac. Salud Pública. 2016; 33(3): 414-424. doi: 10.17533/ udea.rfnsp.v34nla06

11. Rodríguez Hernández JM, Rodríguez Rubiano DP, Corrales Barona JC. Barreras de acceso administrativo a los servicios de salud en población Colombiana, 2013. Ciência \& Saúde Coletiva. 2015; 20(6):19471958. doi: 10.1590/1413-81232015206.12122014

12. Guerrero-Ramírez R, Meneses-La Riva ME, De la Cruz Ruiz M. Cuidado humanizado de enfermería según la teoría de Jean Watson, servicio de medicina del Hospital Daniel Alcides Carrión. Lima-Callao, 2015. Rev. Enferm. Herediana [Internet]. 2016 [citado 1 de mayo del 2018]; 9(2): [aproximadamente 9p.]. Disponible en: https://faenf.cayetano.edu.pe/ images/2017/revistavo19/9.pdf

13. Aldrete-Rodríguez MG, Navarro-Meza C, González-Baltazar R, Contreras-Estrada MI, Pérez-Aldrete J. Factores sicosociales y síndrome de Burnout en personal de enfermería de una unidad de tercer nivel de atención a la salud. Revista Cienc. Trab. [Internet]. 2017 [citado 17 de julio del 2018]; 17 (52): [aproximadamente 4p.]. Disponible en: https://scielo. conicyt.cl/pdf/cyt/v17n52/art07.pdf

14. Bernal-González I, Pedraza-Melo NA, SánchezLimón ML. El clima organizacional y su relación con la calidad de los servicios públicos de salud: diseño de un modelo teórico. Rev. Estudios Generales. 2014; 31(134): 8-19 doi: 10.1016/j.estger.2014.08.003

15. Ayala-García J. La salud en Colombia: más cobertura pero menos acceso [documento]. 2014 [citado 4 de septiembre del 2017]. Disponible en: http:// www.banrep.gov.co/docum/Lectura_finanzas/pdf/ dtser_204.pdf

16. Barragán JA, Pedraza YD, Rincón MR. Calidad percibida de la atención de enfermería por usuarios hospitalizados, Tunja, 2011. Rev. Fac. Nac. Salud Pública [Internet]. 2014 [citado 4 de septiembre del 2017]; 31(2): [aproximadamente 6p.]. Disponible en: http://www.scielo.org.co/pdf/rfnsp/ v31n2/v31n2a04.pdf

17. Ministerio de Salud y Protección Social. Política de atención integral en salud "Un sistema de salud al servicio de la gente". Bogotá: MINSALUD; 2016. 96p.
18. Beltrán-Salazar O. The meaning of humanized nursing care for those participating in it: Importance of efforts of nurses and healthcare institutions. Invest. Educ. Enferm. 2016; 34 (1): 18-28. doi: 10.17533/ udea.iee.v34n1a03

19. Molina G, Ramírez A. Conflicto de valores en el sistema de salud de Colombia: entre la economía de mercado y la normativa constitucional 2007-2009. Rev. Panam. Salud Pública [Internet]. 2014 [citado 4 de septiembre del 2017]; 33(4): [aproximadamente 6p.]. Disponible en: https://www.scielosp.org/pdf/ rpsp/v33n4/a08v33n4.pdf

20. Rubio-Acuña M, Arias-Burgos Ml. Fenomenología y conocimiento disciplinar de enfermería. Rev. Cubana. Enfermer [Internet]. 2013 [citado 24 de febrero del 2017]; 29(3): [aproximadamente 7p]. Disponible en:http://scielo.sld.cu/scielo.php?script=sci_arttext\&pid=S0864-03192013000300005

21. Dúque-Páramo MC, Castellanos-Soriano F, Buitrago-García C. La investigación cualitativa en enfermería en Colombia (1990-2010). Enferm. Glob. [Internet]. 2014 [citado 13 de febrero del 2017]; 13(36): [aproximadamente 14p.] Disponible en: http://www.scielo.br/pdf/tce/v19n4/20.pdf

22. García-Guerra LJ. El código deontológico en enfermería. Comparativa entre el código del CIE, Colombia y España. Bogotá: Chía; 2015.

23. Valderrama-Sanabria ML, Peña-Pita AP, Clavijo Álvarez LM. Narrativa: el estudiante de enfermería aprendiendo el arte de cuidar. Rev. Cuid. 2017; 8(1): 1488-98. doi: 10.15649/cuidarte.v8i1.362

24. Osorio-Castaño JH. Patrón de conocimiento socio-político en enfermería: reflexiones conceptuales. Rev. Cuid. 2016; 7(2): 1352-57. doi: 10.15649/ cuidarte.v7i2.319

25. Gómez-Rojas ML, Rodríguez-Díaz BL. Situación de enfermería como herramienta para enseñar el proceso de atención de enfermería. Rev. Cuid. [Internet]. 2013 [citado 17 de mayo del 2017]. 4(1): [aproximadamente 5p.] Disponible en: http://www. redalyc.org/pdf $/ 3595 / 359533224016 . p d f$

26. Hoyos-Vertel LM. Enfermería Basada en la Evidencia-EBE Blog [Internet]. Bogotá: Luis Miguel Hoyos. 2014 Agosto [citado 30 de marzo del 2016]. Disponible en: http://luismiguelhoyos.blogspot.com.co/2014/08/patrones-de-conocimiento-de-enfermeria.html 